\title{
Genitores potenciais para hibridações identificados por divergência genética em feijão carioca
}

\author{
Bean parents for hybridization identified by genetic divergence in \\ "carioca" bean
}

Nerinéia Dalfollo Ribeiro ${ }^{1}$ Lindolfo Storck $^{2}$

\section{RESUMO}

Noventa genótipos de feijão carioca (Phaseolus vulgaris L.) foram avaliados, em dois anos agrícolas, em Santa Maria, RS, a fim de definir quais características agromorfológicas constituem-se como melhores descritores, realizar agrupamento em função de dissimilaridade genética $e$ de definir quais combinações hibridas mais promissoras serão obtidas para o desenvolvimento de populações segregantes. Dos 20 caracteres agromorfológicos avaliados, apenas nove (ferrugem nos legumes, acamamento, nota geral, cor do tegumento, rendimento de grãos, massa de 100 sementes, altura de inserção do primeiro legume, altura de inserção do último legume e número de sementes por legume) apresentaram maior contribuição para a divergência genética. Os genótipos de feijão carioca foram agrupados pelo método hierárquico de ligação completa. Populações segregantes, com variabilidade genética superior, podem ser obtidas com hibridações entre o genótipo ESAL $550 \mathrm{com}$ genótipos do grupo 2 (LH-6, 17-4-32, R-78, H-4-5 e R-102) elou com genótipos do grupo 3 (FT 97-188, Cati-Taquari, CII-328, Carioca Precoce, FT 97-41, LH-11, FT 91-4067, Iapar 31, CI 102, Carioca MG, CII-54 e R-102).

Palavras-chave: Phaseolus vulgaris, descritores agromorfológicos, agrupamento, variabilidade genética.

\section{ABSTRACT}

Carioca bean genotypes (Phaseolus vulgaris $L$.) were evaluated in two growing seasons in Santa Maria, RS, Brazil. The objectives of this work were to evaluate which morpho-agronomic characteristics were the best descriptors, to group the genotypes in relation to genetic diversity and to determine which hybrid combinations are promissing to obtain higher segregation populations in carioca bean. From the 20 morpho-agronomic characteristics evaluated, only seven (pod rust, lodging, general note, colour of seed tegument, grain yield, 100 seed weight, height of first and final pod insertion and number of seeds per pod) showed higher contribution to genetic diversity. The evaluated carioca bean genotypes were clustered by the complete linkage method. The following hybrid combinations were promissing for obtaining segregant population with higher genetic variability: the genotype ESAL 550 with the genotype of the group 2 (LH-6, 17-4-32, R-78, H-4-5 e R-102) and genotype of the group 3 (FT 97-188, Cati-Taquari, CII-328, Carioca Precoce, FT 97-41, LH-11, FT 91-4067, Iapar 31, CI 102, Carioca MG, CII-54 and R102).

Key words: Phaseolus vulgaris, morpho-agronomic descriptors, clustering, genetic variability.

\section{INTRODUÇÃO}

O feijão (Phaseolus vulgaris L.) tem origem múltipla e sua domesticação ocorreu independentemente, em locais distintos. O tamanho das sementes é a característica marcante no germoplasma que o dividiu em dois grupos: Mesoamérica (sementes pequenas) e Andes (sementes grandes) (McCLEAN et al., 1993).

Atualmente, no Brasil, há um grande número de genótipos com características distintas dos mais variados grupos comerciais (preto, carioca e outros). Embora exista preferência local por determinada coloração de sementes, o feijão carioca tem maior aceitação nacional e é o tipo de grão mais semeado no país. Desse grupo, a cultivar Carioca é responsável pela maioria da produção de grãos de feijão e qualquer nova cultivar deverá ter características

${ }^{1}$ Engenheiro Agrônomo, Doutor, Professor Adjunto, Departamento de Fitotecnia, Universidade Federal de Santa Maria (UFSM), 97105-900. Santa Maria, RS. E-mail: nerineia@ccr.ufsm.br. Autor para correspondência.

${ }^{2}$ Engenheiro Agrônomo, Doutor, Professor Titular, Departamento de Fitotecnia, UFSM. Bolsista do CNPq. 
de grãos semelhantes às da 'Carioca', para aumentar a sua probabilidade de aceitação por produtores e consumidores (ABREU et al., 1994). Por isso, esta foi muito utilizada como genitor em grande número de cruzamentos, até 1996, quando houve o lançamento da 'Pérola', gerando germoplasma aparentado (RIBEIRO, 2001). Além disso, sabe-se que a utilização de genitores não divergentes ou similares reduz as chances de obtenção de progressos na seleção, pois, além da perda de tempo com hibridações e condução de populações segregantes a campo, diminui a probabilidade de desenvolver uma cultivar superior, com características competitivas, para um mercado cada vez mais exigente.

Como o melhoramento do feijoeiro baseiase, principalmente, na hibridação de cultivares e linhagens, gerando populações segregantes, em que se procede à seleção de linhagens superiores, tornase importante o conhecimento da dissimilaridade genética entre os genitores. Em vista disso, estudos sobre divergência genética são de grande importância em programas de melhoramento, pois fornecem parâmetros para a identificação de genitores que, quando cruzados, possibilitam maior probabilidade de recuperar genótipos superiores nas gerações segregantes (CRUZ \& REGAZZI, 1997).

A divergência genética tem sido avaliada por meio de técnicas biométricas, baseadas na quantificação da heterose, ou por processos preditivos. Dentro dos métodos fundamentados em modelos biométricos, cita-se a análise dialélica, na qual faz-se necessária a avaliação de 'p' genitores e de todas as suas combinações híbridas possíveis ( $\mathrm{p}(\mathrm{p}$ 1)/2) (CRUZ \& REGAZZI, 1997). Assim, quando o valor de 'p' é elevado, a obtenção de material experimental pode ser impraticável e o estudo inviabilizado. Em feijão, associa-se ainda a hibridação manual controlada, técnica bastante meticulosa e dependente de vários fatores ambientais, além de habilidades manuais do operador.

Os métodos preditivos, por dispensarem a obtenção prévia das combinações híbridas, têm merecido considerável ênfase na cultura do feijoeiro. Esses métodos consideram as diferenças morfológicas, fisiológicas, entre outras, apresentadas pelos genitores na determinação da divergência genética (FONSECA \& SILVA, 1997; COIMBRA \& CARVALHO, 1999; COIMBRA et al., 1999; FONSECA \& SILVA, 1999; COIMBRA et al., 2000; MACHADO et al., 2000).

Diante desses fatos, foram objetivos desse trabalho: determinar quais as características agromorfológicas constituem-se como descritores para genótipos de feijão carioca; agrupar os genótipos em grupos de dissimilaridade genética, através do método hierárquico de ligação completa; definir as combinações híbridas mais promissoras para o desenvolvimento de populações segregantes em feijão carioca.

\section{MATERIAL E MÉTODOS}

Os experimentos foram conduzidos em área do Departamento de Fitotecnia da Universidade Federal de Santa Maria, nos anos agrícolas 1998/99 e 1999/00, em solo da Unidade de Mapeamento Santa Maria (Brunizem Hidromórfico).

Os tratamentos consistiram de 90 genótipos de feijão carioca, sendo os genótipos com genealogia conhecida identificados na tabela 1. Para a escolha dos genótipos considerou-se a utilização das cultivares registradas para a semeadura e das linhagens homozigotas promissoras, provenientes de vários programas de melhoramento no Brasil.

O delineamento experimental utilizado foi blocos casualizados, com duas repetições. No primeiro ano, as parcelas foram compostas de uma fileira com $5 \mathrm{~m}$ de comprimento, espaçadas de $0,50 \mathrm{~m}$, e com área útil de $2,5 \mathrm{~m}^{2}$. A semeadura foi realizada em 4/11/1998 e com densidade de 10 sementes por metro. No segundo ano agrícola, usaram-se parcelas compostas de duas fileiras de $3 \mathrm{~m}$ de comprimento, espaçadas de $0,50 \mathrm{~m}$, e área útil de $3 \mathrm{~m}^{2}$. A semeadura foi em $27 / 10 / 1999$, e a densidade foi ajustada de acordo com os diferentes hábitos de crescimento dos genótipos (CEPEF, 2000). O preparo do solo foi realizado de forma convencional e as adubações foram baseadas na análise química do solo, seguindo as Recomendações de Adubação e Calagem para os Estados do Rio Grande do Sul e Santa Catarina (ROLAS, 1995).

O controle de insetos foi efetuado com a aplicação de metamidofós $\left(750 \mathrm{~mL} \mathrm{ha}^{-1}\right) \mathrm{e}$, para a eliminação de plantas invasoras usou-se o herbicida fusilade $\left(850 \mathrm{~mL} \mathrm{ha}^{-1}\right)$ e arranquio manual de plantas remanecentes. Os tratos culturais e a irrigação foram realizados sempre que necessário, de forma a não prejudicar o desenvolvimento normal da cultura. Não se fez o controle de moléstias a fim de se verificar a ocorrência de patógenos nos genótipos.

Os caracteres agromorfológicos avaliados foram: estande inicial, antracnose (Colletotrichum lindemuthianum), crestamento bacteriano comum (Xanthomonas campestris pv. phaseoli) e ferrugem (Uromyces phaseoli var. typica) nas folhas e nos legumes (por ocasião da floração e da maturação fisiológica, respectivamente), acamamento, nota geral de adaptação, maturação de colheita, ciclo cultural e 
Tabela 1 - Hábito de crescimento (HC), massa de 100 sementes (MCS) e instituição de procedência dos genótipos de feijão carioca com genealogia conhecida. Santa Maria, UFSM, 2000.

\begin{tabular}{clllll}
\hline $\mathrm{N}^{\text {. }}$ & Genótipo & HC* & MCS(g) & Genealogia** & Procedência*** \\
\hline 4 & Iapar 31 & II & 24,9 & BAC 4/RAI 46//BAC 2/IGUAÇÚ/3/BAT/BAC 4 & IAPAR \\
11 & BR IPA-11 Brigida & II & 23,0 & Rio Tibagi / Carioca // Carioca / Rio Tibagi) & CNPAF \\
12 & IAC Akytã & II & 21,6 & DOR 41 x (10-3-1 . TU1R-2 . 10-9-1) & IAC \\
13 & TPS Bonito & II & 20,3 & Iapar 14 / IAC Carioca & TPS \\
26 & Iapar 72 & III & 26,0 & Phaseolus vulgaris (cv.Carioca) / P. Coccineus & IAPAR \\
30 & Carioca MG & II & 18,0 & Carioca 80 / Rio Tibagi & CNPAF \\
31 & Iapar 57 & II & 24,5 & Porrillo Sintético/Aeté 1-38 // Cena 83-1/ 3/ Iapar-BAC 32 & IAPAR \\
32 & Iapar 80 & II & - & [(A248/EMP 117)] // \{[BAT 93/ (Carioca 99/ GN Nebraska \# 27/ IAPAR & IAPA \\
& & & & Sel Aroana)] & IAC \\
33 & IAC Carioca Aruã & II/III & 25,0 & Cornell 49-242/ AB-136 & IAPAR \\
45 & Iapar 81 & III & - & IAPAR BAC 38 / MD 648 // GF 3721 / IAPAR BAC 25 & IAC \\
46 & IAC Pyatã & II & 25,5 & DOR 41 x (10-3-1 . TU1R-2 . 10-9-1) & CNPAF \\
49 & Goytacazes & III & - & A 106 / A 63 & CNPAF \\
50 & Princesa & II & 23,0 & A-252 x BAT 1550 & CNPAF \\
52 & Rudá & II/III & 19,4 & Carioca / Rio Tibagi & TPS \\
58 & FT 91 - 4044 & III & - & FT 86-134/BULK 20 & CNPAF \\
63 & Pérola & II/III & 27,0 & Seleção Massal na cultivar Aporé & IAC \\
65 & Carioca & III & 25,4 & Seleção em cultivar local em São Paulo & CNPAF \\
76 & Aporé & III & 27,0 & Carioca/México 168 // Carioca/Bat 76 & UFLA \\
89 & ESAL 693 & I & 19,0 & Carioca TU / Linha 3272 & \\
\hline
\end{tabular}

* I: hábito de crescimento determinado; II: hábito de crescimento indeterminado com guias curtas; III: hábito de crescimento indeterminado com guias longas.

** /: cruzamento simples; //: cruzamento duplo; + dado do experimento.

*** TPS: Terassawa Produção de Sementes, Paraná; IAC: Instituto Agronômico de Campinas, São Paulo; IAPAR: Instituto Agronômico do Paraná, Paraná; CNPAF: Centro Nacional de Pesquisa de Arroz e Feijão, Goiás; UFLA: Universidade Federal de Lavras, Minas Gerais.

estande final. Essas avaliações foram baseadas nos estádios de crescimento da cultura segundo a escala proposta pelo CIAT (1987). O rendimento de grãos a $13 \%$ de umidade e a cor do tegumento das sementes, através do sistema $\mathrm{L}-\mathrm{a}-\mathrm{b}$, com um colorímetro, marca Minolta, modelo CR-310, foram obtidos logo após a colheita. O eixo vertical ' $L$ ' avalia a claridade da cor do tegumento da amostra, variando do preto ao branco, o eixo ' $a$ ' da cor verde ao vermelho e o eixo ' $b$ ' da cor azul ao amarelo. Para o feijão carioca, importa a cor clara do tegumento das sementes, razão pela qual foram apresentados apenas os valores 'L'. As avaliações baseadas na média de cinco plantas coletadas ao acaso, na parcela útil, foram: altura de inserção do primeiro legume, altura de inserção do último legume, número de legumes por planta, número de sementes por planta, número de sementes por legume e massa de 100 sementes.

Os dados foram submetidos à análise da variância conjunta (90 genótipos, dois anos e dois blocos por ano), considerando anos, blocos, interação ano $\mathrm{X}$ genótipo e erro como sendo de efeito aleatório e genótipo de efeito fixo. As médias de genótipos foram comparadas pelo teste de Skott-Knott (RAMALHO et al., 2000). Com a utilização das matrizes das médias, das variâncias e covariâncias residuais das variáveis mais importantes para a divergência genética, procederam-se os cálculos das distâncias de Mahalanobis entre todos os pares de genótipos, sendo o agrupamento realizado pelo método hierárquico de ligação completa (CRUZ \& REGAZZI, 1997; CRUZ, 2001).

\section{RESULTADOS E DISCUSSÃO}

O resumo das análises da variância, médias e coeficiente de variação para os 20 caracteres agromorfológicos estudados, envolvendo os 90 genótipos de feijão carioca, encontra-se na tabela 2 . Diferenças significativas, entre genótipos, ocorreram em relação à maioria das características, exceto para moléstias (antracnose, crestamento bacteriano comum e ferrugem nas folhas), estande inicial e final, maturação e ciclo cultural e número de legumes e de sementes 
Tabela 2 - Resumo das análises de variância dos 20 caracteres agromorfológicos avaliados em 90 genótipos de feijão carioca. Santa Maria, UFSM, 2000.

\begin{tabular}{|c|c|c|c|c|c|c|}
\hline \multirow[b]{2}{*}{ Caracteres } & \multicolumn{4}{|c|}{ Quadrados Médios } & \multirow[b]{2}{*}{ Média } & \multirow[b]{2}{*}{$\mathrm{CV}(\%)$} \\
\hline & $\begin{array}{c}\text { Ano + Bloco } \\
\text { (Ano) }\end{array}$ & Genótipos & $\begin{array}{c}\text { Ano x } \\
\text { Genótipos }\end{array}$ & Erro & & \\
\hline Graus de liberdade & 3 & 89 & 89 & 178 & - & - \\
\hline Antracnose nas folhas & 0,00 & $0,00^{\mathrm{ns}}$ & 0,00 & 0,00 & 1,00 & 0,0 \\
\hline Antracnose nos legumes & $0,16^{*}$ & $0,04^{\mathrm{ns}}$ & $0,04^{\mathrm{ns}}$ & 0,037 & 1,02 & 18,7 \\
\hline Crest. Bact. nas folhas & $0,13^{\mathrm{ns}}$ & $0,05^{\mathrm{ns}}$ & $0,06^{\mathrm{ns}}$ & 0,064 & 1,04 & 24,2 \\
\hline Crest. Bact. nos legumes & $0,22^{\mathrm{ns}}$ & $0,21^{\mathrm{ns}}$ & $0,22 *$ & 0,126 & 1,05 & 33,8 \\
\hline Ferrugem nas folhas & $0,00^{\mathrm{ns}}$ & $0,00^{\mathrm{ns}}$ & $0,003^{\mathrm{ns}}$ & 0,003 & 1,00 & 5,2 \\
\hline Ferrugem nos legumes & $3,40^{*}$ & $0,42 *$ & $0,24^{\mathrm{ns}}$ & 0,286 & 1,19 & 44,9 \\
\hline Acamamento & $11,54 *$ & $3,62^{*}$ & $2,20^{\mathrm{ns}}$ & 1,625 & 4,29 & 29,7 \\
\hline Nota geral & $17,8^{*}$ & $9,30^{*}$ & $4,20^{\mathrm{ns}}$ & 3,213 & 5,68 & 31,5 \\
\hline Estande inicial & $5722 *$ & $62,60^{\mathrm{ns}}$ & $81,8^{*}$ & 30,74 & 50,06 & 11,1 \\
\hline Estande final & $983^{*}$ & $95,6^{\mathrm{ns}}$ & $105,2 *$ & 56,2 & 44,31 & 16,9 \\
\hline Maturação de colheita (dias) & $4589 *$ & $20,9^{\mathrm{ns}}$ & $22,2^{*}$ & 6,58 & 81,05 & 3,1 \\
\hline Ciclo cultural (dias) & $2326^{*}$ & $13,9^{\mathrm{ns}}$ & $13,3^{*}$ & 2,29 & 85,58 & 1,7 \\
\hline Cor do tegumento ("L") & $430,1^{*}$ & $19,4^{*}$ & $10,2 *$ & 0,87 & 54,50 & 1,7 \\
\hline Rendimento de grãos $\left(\mathrm{t} \mathrm{ha}^{-1}\right)$ & $27,560^{*}$ & $0,710^{*}$ & $0,310^{\mathrm{ns}}$ & 0,286 & 1,802 & 29,7 \\
\hline Massa de 100 sementes (g) & $385,2 *$ & $27,3^{*}$ & $7,6^{*}$ & 5,64 & 21,09 & 10,7 \\
\hline Alt. ins. $1^{\circ}$ legume $(\mathrm{cm})$ & $460,3^{*}$ & $23,9^{*}$ & $14,5^{*}$ & 7,59 & 12,49 & 22,1 \\
\hline Alt. ins. último legume $(\mathrm{cm})$ & $3597 *$ & $181,7^{*}$ & $96,1^{*}$ & 56,10 & 42,36 & 17,7 \\
\hline $\mathrm{N}^{\mathrm{o}}$ de legumes por planta & $334,1^{*}$ & $18,2^{\mathrm{ns}}$ & $16,4^{*}$ & 11,25 & 12,79 & 26,2 \\
\hline $\mathrm{N}^{\mathrm{o}}$ de sementes por planta & $13440^{*}$ & $607^{\mathrm{ns}}$ & $520^{*}$ & 308,7 & 63,95 & 27,5 \\
\hline $\mathrm{N}^{\mathrm{o}}$ de sementes por legume & $0,91^{\mathrm{ns}}$ & $1,09^{*}$ & $0,75^{*}$ & 0,525 & 5,00 & 14,5 \\
\hline
\end{tabular}

ns: Não significativo. * significativo em nível de 5\% de probabilidade de erro pelo teste de $\mathrm{F}$.

por planta. A não observância de diferenças significativas para moléstias deve-se ao fato de que, praticamente, não houve incidência de moléstias nos dois anos, pois as condições ambientais não foram propícias a sua ocorrência. Com relação ao estande inicial e final, observou-se homogeneidade para número de plantas, indicando uma boa emergência e pouca $(11,1 \%)$ perda de plantas durante o desenvolvimento das plantas.

$\mathrm{O}$ fato da interação ano $\mathrm{X}$ genótipo ser significativa é prejudicial para o planejamento das hibridações e para a seleção de genótipos superiores. Mas, se os ensaios fossem realizados em um único ano, em quatro blocos, também haveria interação blocos X genótipos (estimado pelo erro experimental) e as médias e as medidas de dissimilaridades teriam validade apenas para aquele ano. Assim, na média de dois anos, os resultados devem ser mais representativos para o planejamento das hibridações, mesmo com interação significativa.
Para identificar os caracteres importantes para a discriminação da variabilidade genética entre os genótipos, considerou-se a exclusão de caracteres em que não houve efeito significativo para genótipos. Assim, dos vinte descritores agromorfológicos avaliados, apenas nove, os da tabela 3 , foram utilizados para a estimação das dissimilaridades e para o agrupamento dos genótipos (Figura 1).

As médias dos caracteres agromorfológicos que foram usados para a análise de agrupamento, comparados pelo método de Skott-Knott, estão apresentados na tabela 3 . O rendimento médio dos genótipos foi de $1.802 \mathrm{~kg} \mathrm{ha}^{-1}$. Se considerarmos o fato de que apenas a 'TPS Bonito' está registrada para o cultivo no Rio Grande do Sul, para a safra 2000/01 (CEPEF, 2000), sendo, portanto, a única testemunha para esse grupo de grãos, verifica-se que 86 dos 90 genótipos a superaram em rendimento de grãos, em Santa Maria, nos anos agrícolas 1998/99 e 1999/00. A linhagem FT 97-188 superou em 290\% a 'TPS Bonito'

Ciência Rural, v. 33, n. 3, mai-jun, 2003. 
Tabela 3 - Médias da ferrugem nos legumes (FEV), acamamento (ACA), nota geral (NG), coloração do tegumento das sementes (COR), rendimento de grãos (REND), massa de 100 sementes (MCS), altura de inserção de primeiro legume (APL), altura de inserção de último legume (AUL) e número de sementes por legume (SPL) referentes a 90 genótipos de feijão carioca. Santa Maria, UFSM, 2000 .

\begin{tabular}{|c|c|c|c|c|c|c|c|c|c|c|}
\hline $\mathrm{N}^{\mathrm{o}}$ & Genótipo & FEV & ACA & NG & COR & $\begin{array}{c}\text { REND } \\
\left(\mathrm{kg}^{-h^{-1}}\right)\end{array}$ & $\operatorname{MCS}(\mathrm{g})$ & APL (cm) & AUL (cm) & SPL \\
\hline 1 & FT 91-3037 & $1,00 \mathrm{E}$ & $4,2 \mathrm{~A}$ & $3,0 \mathrm{E}$ & $54,87 \mathrm{~A}$ & $1,810 \mathrm{E}$ & $17,86 \mathrm{H}$ & $10,5 \mathrm{G}$ & $40,5 \mathrm{D}$ & $4,82 \mathrm{E}$ \\
\hline 2 & Iapar 14 & $1,50 \mathrm{D}$ & $5,0 \mathrm{~A}$ & $6,7 \mathrm{D}$ & $55,71 \mathrm{~A}$ & $1,486 \mathrm{~F}$ & $16,14 \mathrm{H}$ & $15,6 \mathrm{C}$ & $44,4 \mathrm{C}$ & $5,47 \mathrm{C}$ \\
\hline 3 & FT 97-144 & $1,00 \mathrm{E}$ & $2,0 \mathrm{~B}$ & $2,2 \mathrm{E}$ & $52,02 \mathrm{~B}$ & $1,681 \mathrm{E}$ & $23,06 \mathrm{~A}$ & $10,7 \mathrm{G}$ & $43,0 \mathrm{C}$ & $4,65 \mathrm{E}$ \\
\hline 4 & Iapar 31 & $1,50 \mathrm{D}$ & $3,0 \mathrm{~B}$ & $3,2 \mathrm{E}$ & $52,92 \mathrm{~B}$ & $2,365 \mathrm{C}$ & $22,95 \mathrm{~A}$ & $8,5 \mathrm{G}$ & $34,9 \mathrm{G}$ & $5,60 \mathrm{C}$ \\
\hline 5 & $\mathrm{R}-78$ & $1,00 \mathrm{E}$ & $5,7 \mathrm{~A}$ & $6,5 \mathrm{D}$ & $60,00 \mathrm{~A}$ & $1,691 \mathrm{E}$ & $20,45 \mathrm{~F}$ & $10,5 \mathrm{G}$ & $32,5 \mathrm{G}$ & $4,95 \mathrm{D}$ \\
\hline 6 & FT 97-23 & $1,00 \mathrm{E}$ & $4,2 \mathrm{~A}$ & $6,0 \mathrm{D}$ & $53,24 \mathrm{~B}$ & $1,405 \mathrm{G}$ & $21,65 \mathrm{~A}$ & $10,1 \mathrm{G}$ & $40,7 \mathrm{D}$ & $5,57 \mathrm{C}$ \\
\hline 7 & IAC Carioca & $1,25 \mathrm{E}$ & $6,0 \mathrm{~A}$ & $7,2 \mathrm{C}$ & $55,48 \mathrm{~A}$ & $1,396 \mathrm{G}$ & $18,71 \mathrm{G}$ & $15,4 \mathrm{C}$ & $54,3 \mathrm{~B}$ & $4,87 \mathrm{E}$ \\
\hline 8 & CII-299 & $1,00 \mathrm{E}$ & $2,2 \mathrm{~B}$ & $2,5 \mathrm{E}$ & $52,80 \mathrm{~B}$ & $1,813 \mathrm{E}$ & $18,85 \mathrm{G}$ & $10,0 \mathrm{G}$ & $40,8 \mathrm{D}$ & $5,30 \mathrm{D}$ \\
\hline 9 & ESAL 696 & $1,00 \mathrm{E}$ & $5,0 \mathrm{~A}$ & $3,2 \mathrm{E}$ & $48,38 \mathrm{~B}$ & $1,163 \mathrm{G}$ & $17,48 \mathrm{H}$ & $11,8 \mathrm{~F}$ & $40,8 \mathrm{D}$ & $3,75 \mathrm{E}$ \\
\hline 10 & R-290 & $1,00 \mathrm{E}$ & $5,0 \mathrm{~A}$ & $7,5 \mathrm{C}$ & $53,10 \mathrm{~B}$ & $1,943 \mathrm{D}$ & $23,72 \mathrm{~A}$ & $9,6 \mathrm{G}$ & $35,2 \mathrm{G}$ & $4,70 \mathrm{E}$ \\
\hline 11 & BR IPA-11 Brigida & $1,25 \mathrm{E}$ & $3,7 \mathrm{~B}$ & $5,5 \mathrm{E}$ & $49,38 \mathrm{~B}$ & $2,159 \mathrm{D}$ & 19,19G & $9,5 \mathrm{G}$ & $40,2 \mathrm{D}$ & $5,47 \mathrm{C}$ \\
\hline 12 & IAC Akytã & $1,00 \mathrm{E}$ & $3,2 \mathrm{~B}$ & $4,5 \mathrm{E}$ & $53,76 \mathrm{~B}$ & $1,459 \mathrm{~F}$ & $15,35 \mathrm{H}$ & $10,3 \mathrm{G}$ & $36,8 \mathrm{~F}$ & $5,02 \mathrm{D}$ \\
\hline 13 & TPS Bonito & $1,00 \mathrm{E}$ & $5,7 \mathrm{~A}$ & $7,0 \mathrm{D}$ & $54,50 \mathrm{~B}$ & $1,056 \mathrm{G}$ & $16,39 \mathrm{H}$ & $20,6 \mathrm{~A}$ & $46,2 \mathrm{C}$ & $4,67 \mathrm{E}$ \\
\hline 14 & ESAL 695 & $1,75 \mathrm{C}$ & $4,2 \mathrm{~A}$ & $3,5 \mathrm{E}$ & $54,10 \mathrm{~B}$ & $1,721 \mathrm{E}$ & $21,79 \mathrm{~A}$ & $10,8 \mathrm{G}$ & $35,7 \mathrm{G}$ & $5,00 \mathrm{D}$ \\
\hline 15 & LH-11 & $1,00 \mathrm{E}$ & $3,7 \mathrm{~B}$ & $5,5 \mathrm{E}$ & $55,50 \mathrm{~A}$ & $2,440 \mathrm{C}$ & $24,13 \mathrm{~A}$ & $13,9 \mathrm{D}$ & $47,2 \mathrm{C}$ & $4,92 \mathrm{D}$ \\
\hline 16 & CI-107-5 & $1,00 \mathrm{E}$ & $4,2 \mathrm{~A}$ & $7,7 \mathrm{C}$ & $56,25 \mathrm{~A}$ & $2,217 \mathrm{D}$ & $22,60 \mathrm{~A}$ & $9,9 \mathrm{G}$ & $35,0 \mathrm{G}$ & $4,52 \mathrm{E}$ \\
\hline 17 & CII-53 & $1,00 \mathrm{E}$ & $5,2 \mathrm{~A}$ & $5,2 \mathrm{E}$ & $53,53 \mathrm{~B}$ & $1,342 \mathrm{G}$ & $15,92 \mathrm{H}$ & $14,2 \mathrm{D}$ & $55,1 \mathrm{~B}$ & $5,77 \mathrm{C}$ \\
\hline 18 & R-175 & $1,00 \mathrm{E}$ & $4,2 \mathrm{~A}$ & $6,2 \mathrm{D}$ & $55,96 \mathrm{~A}$ & $2,089 \mathrm{D}$ & $23,62 \mathrm{~A}$ & $11,3 \mathrm{~F}$ & $35,6 \mathrm{G}$ & $5,10 \mathrm{D}$ \\
\hline 19 & FT 97-115 & $1,00 \mathrm{E}$ & $6,0 \mathrm{~A}$ & $7,0 \mathrm{D}$ & $52,20 \mathrm{~B}$ & $1,368 \mathrm{G}$ & $17,52 \mathrm{H}$ & $14,9 \mathrm{D}$ & $46,1 \mathrm{C}$ & $4,87 \mathrm{E}$ \\
\hline 20 & LH-1 & $1,00 \mathrm{E}$ & $3,7 \mathrm{~B}$ & $4,2 \mathrm{E}$ & $53,36 \mathrm{~B}$ & $1,936 \mathrm{D}$ & $18,91 \mathrm{G}$ & $11,9 \mathrm{~F}$ & $34,8 \mathrm{G}$ & $5,52 \mathrm{C}$ \\
\hline 21 & $\mathrm{R}-244$ & $1,25 \mathrm{E}$ & $4,5 \mathrm{~A}$ & $6,5 \mathrm{D}$ & $56,88 \mathrm{~A}$ & $1,806 \mathrm{E}$ & $21,85 \mathrm{~A}$ & $10,6 \mathrm{G}$ & $39,4 \mathrm{E}$ & $5,07 \mathrm{D}$ \\
\hline 22 & CII-71 & $1,50 \mathrm{D}$ & $3,5 \mathrm{~B}$ & $5,7 \mathrm{E}$ & $54,12 \mathrm{~B}$ & $2,177 \mathrm{D}$ & $22,55 \mathrm{~A}$ & $13,0 \mathrm{D}$ & $49,0 \mathrm{C}$ & $5,10 \mathrm{D}$ \\
\hline 23 & FT 97-159 & $1,00 \mathrm{E}$ & $2,7 \mathrm{~B}$ & $4,5 \mathrm{E}$ & $53,50 \mathrm{~B}$ & $1,921 \mathrm{D}$ & $22,23 \mathrm{~A}$ & $11,0 \mathrm{~F}$ & $45,1 \mathrm{C}$ & $4,92 \mathrm{D}$ \\
\hline 24 & FT 97-155 & $1,50 \mathrm{D}$ & $3,7 \mathrm{~B}$ & $5,5 \mathrm{E}$ & $53,05 \mathrm{~B}$ & $2,023 \mathrm{D}$ & $20,99 \mathrm{D}$ & $10,2 \mathrm{G}$ & $47,3 \mathrm{C}$ & $5,35 \mathrm{D}$ \\
\hline 25 & CII-244 & $1,00 \mathrm{E}$ & $5,0 \mathrm{~A}$ & $6,7 \mathrm{D}$ & $53,50 \mathrm{~B}$ & 1,969D & $20,84 \mathrm{E}$ & $13,5 \mathrm{D}$ & $42,4 \mathrm{C}$ & $4,92 \mathrm{D}$ \\
\hline 26 & Iapar 72 & $1,50 \mathrm{D}$ & $5,5 \mathrm{~A}$ & $8,2 \mathrm{~A}$ & $53,26 \mathrm{~B}$ & $1,927 \mathrm{D}$ & $21,28 \mathrm{~A}$ & $12,2 \mathrm{~F}$ & $40,6 \mathrm{D}$ & $4,67 \mathrm{E}$ \\
\hline 27 & FT 97-175 & $1,25 \mathrm{E}$ & $4,2 \mathrm{~A}$ & $6,7 \mathrm{D}$ & $53,47 \mathrm{~B}$ & $2,227 \mathrm{D}$ & $21,08 \mathrm{C}$ & $10,9 \mathrm{G}$ & $45,2 \mathrm{C}$ & $5,65 \mathrm{C}$ \\
\hline 28 & CII-90 & $1,00 \mathrm{E}$ & $3,5 \mathrm{~B}$ & $5,0 \mathrm{E}$ & $50,25 \mathrm{~B}$ & $1,984 \mathrm{D}$ & $20,43 \mathrm{~F}$ & $13,7 \mathrm{D}$ & $47,6 \mathrm{C}$ & $5,25 \mathrm{D}$ \\
\hline 29 & LH-2 & $1,00 \mathrm{E}$ & $5,0 \mathrm{~A}$ & $6,5 \mathrm{D}$ & $54,19 \mathrm{~B}$ & $1,886 \mathrm{D}$ & $20,05 \mathrm{~F}$ & $12,9 \mathrm{D}$ & $44,9 \mathrm{C}$ & $5,15 \mathrm{D}$ \\
\hline 30 & Carioca MG & $1,00 \mathrm{E}$ & $5,2 \mathrm{~A}$ & $7,0 \mathrm{D}$ & $51,34 \mathrm{~B}$ & $2,328 \mathrm{C}$ & $20,37 \mathrm{~F}$ & $12,6 \mathrm{E}$ & $43,4 \mathrm{C}$ & $5,80 \mathrm{C}$ \\
\hline 31 & Iapar 57 & $1,00 \mathrm{E}$ & $5,0 \mathrm{~A}$ & $5,5 \mathrm{E}$ & $56,67 \mathrm{~A}$ & $1,975 \mathrm{D}$ & $22,86 \mathrm{~A}$ & $8,9 \mathrm{G}$ & $30,0 \mathrm{G}$ & $4,77 \mathrm{E}$ \\
\hline 32 & Iapar 80 & $1,00 \mathrm{E}$ & $3,2 \mathrm{~B}$ & $5,5 \mathrm{E}$ & $53,00 \mathrm{~B}$ & $2,122 \mathrm{D}$ & $21,46 \mathrm{~A}$ & $13,0 \mathrm{D}$ & $44,3 \mathrm{C}$ & $6,02 \mathrm{~B}$ \\
\hline 33 & IAC Carioca Aruã & $2,50 \mathrm{~B}$ & $3,0 \mathrm{~B}$ & $5,0 \mathrm{E}$ & $57,00 \mathrm{~A}$ & $1,450 \mathrm{~F}$ & $19,79 \mathrm{~F}$ & $12,0 \mathrm{~F}$ & $48,4 \mathrm{C}$ & $4,67 \mathrm{E}$ \\
\hline 34 & FT 97-188 & $1,00 \mathrm{E}$ & $2,2 \mathrm{~B}$ & $3,0 \mathrm{E}$ & $55,94 \mathrm{~A}$ & $3,066 \mathrm{~A}$ & $23,60 \mathrm{~A}$ & $11,5 \mathrm{~F}$ & $48,1 \mathrm{C}$ & $5,42 \mathrm{C}$ \\
\hline 35 & CII-328 & $1,00 \mathrm{E}$ & $5,0 \mathrm{~A}$ & $6,2 \mathrm{D}$ & $56,60 \mathrm{~A}$ & $2,545 \mathrm{C}$ & $23,73 \mathrm{~A}$ & $14,8 \mathrm{D}$ & $54,2 \mathrm{~B}$ & $4,65 \mathrm{E}$ \\
\hline 36 & FT 97-124 & $1,00 \mathrm{E}$ & $4,5 \mathrm{~A}$ & $5,2 \mathrm{E}$ & $54,86 \mathrm{~A}$ & $1,762 \mathrm{E}$ & $19,90 \mathrm{~F}$ & $10,4 \mathrm{G}$ & $27,9 \mathrm{G}$ & $5,22 \mathrm{D}$ \\
\hline 37 & Cati-Taquari & $1,00 \mathrm{E}$ & $3,5 \mathrm{~B}$ & $6,5 \mathrm{D}$ & $52,88 \mathrm{~B}$ & $2,838 \mathrm{~B}$ & $22,82 \mathrm{~A}$ & $11,9 \mathrm{~F}$ & $41,5 \mathrm{D}$ & $5,82 \mathrm{C}$ \\
\hline 38 & FT 97-119 & $1,25 \mathrm{E}$ & $4,7 \mathrm{~A}$ & $3,5 \mathrm{E}$ & $54,05 \mathrm{~B}$ & $1,417 \mathrm{G}$ & $20,36 \mathrm{~F}$ & $10,8 \mathrm{G}$ & $37,7 \mathrm{~F}$ & $5,12 \mathrm{D}$ \\
\hline 39 & CII-43 & $1,00 \mathrm{E}$ & $4,2 \mathrm{~A}$ & $8,2 \mathrm{~A}$ & $55,64 \mathrm{~A}$ & $2,238 \mathrm{D}$ & $21,40 \mathrm{~A}$ & $9,5 \mathrm{G}$ & $38,6 \mathrm{~F}$ & $5,00 \mathrm{D}$ \\
\hline 40 & ESAL 694 & $1,00 \mathrm{E}$ & $4,0 \mathrm{~B}$ & $3,7 \mathrm{E}$ & $54,08 \mathrm{~B}$ & $1,672 \mathrm{E}$ & $19,99 \mathrm{~F}$ & $8,5 \mathrm{G}$ & $28,0 \mathrm{G}$ & $3,50 \mathrm{E}$ \\
\hline 41 & FT 97-117 & $1,25 \mathrm{E}$ & $4,0 \mathrm{~B}$ & $7,2 \mathrm{C}$ & $55,43 \mathrm{~A}$ & $2,008 \mathrm{D}$ & $21,58 \mathrm{~A}$ & $15,4 \mathrm{C}$ & $44,9 \mathrm{C}$ & $5,37 \mathrm{D}$ \\
\hline 42 & ANPAT 8-12 & $1,00 \mathrm{E}$ & $5,0 \mathrm{~A}$ & $7,5 \mathrm{C}$ & $56,54 \mathrm{~A}$ & $1,468 \mathrm{~F}$ & $19,57 \mathrm{G}$ & $13,7 \mathrm{D}$ & $38,4 \mathrm{~F}$ & $4,57 \mathrm{E}$ \\
\hline 43 & CII-281 & $1,50 \mathrm{D}$ & $5,2 \mathrm{~A}$ & $7,0 \mathrm{D}$ & $54,56 \mathrm{~B}$ & $1,841 \mathrm{D}$ & $22,31 \mathrm{~A}$ & $13,1 \mathrm{D}$ & $37,5 \mathrm{~F}$ & $4,97 \mathrm{D}$ \\
\hline 44 & CII-340 & $1,00 \mathrm{E}$ & $4,5 \mathrm{~A}$ & $5,7 \mathrm{E}$ & $52,34 \mathrm{~B}$ & $1,649 \mathrm{E}$ & $18,16 \mathrm{H}$ & $9,9 \mathrm{G}$ & $40,1 \mathrm{D}$ & $4,77 \mathrm{E}$ \\
\hline 45 & Iapar 81 & $1,50 \mathrm{D}$ & $3,5 \mathrm{~B}$ & $4,0 \mathrm{E}$ & $56,24 \mathrm{~A}$ & $1,477 \mathrm{~F}$ & $22,70 \mathrm{~A}$ & $13,3 \mathrm{D}$ & $37,4 \mathrm{~F}$ & $4,50 \mathrm{E}$ \\
\hline
\end{tabular}

Ciência Rural, v. 33, n. 3, mai-jun, 2003. 


\begin{tabular}{|c|c|c|c|c|c|c|c|c|c|c|}
\hline 46 & IAC Pyatã & $1,00 \mathrm{E}$ & $4,2 \mathrm{~A}$ & $5,5 \mathrm{E}$ & $53,57 \mathrm{~B}$ & $1,554 \mathrm{~F}$ & $17,69 \mathrm{H}$ & $13,6 \mathrm{D}$ & $48,9 \mathrm{C}$ & $5,22 \mathrm{D}$ \\
\hline 47 & CII-54 & $1,00 \mathrm{E}$ & $4,2 \mathrm{~A}$ & $8,0 \mathrm{~B}$ & $56,33 \mathrm{~A}$ & $2,293 \mathrm{C}$ & $20,95 \mathrm{E}$ & $12,3 \mathrm{~F}$ & $38,0 \mathrm{~F}$ & $5,62 \mathrm{C}$ \\
\hline 48 & $\mathrm{H}-4-5$ & $1,00 \mathrm{E}$ & $5,2 \mathrm{~A}$ & $5,5 \mathrm{E}$ & $59,22 \mathrm{~A}$ & $1,813 \mathrm{E}$ & $22,00 \mathrm{~A}$ & $10,2 \mathrm{G}$ & $33,6 \mathrm{G}$ & $4,72 \mathrm{E}$ \\
\hline 49 & Goytacazes & $1,00 \mathrm{E}$ & $4,7 \mathrm{~A}$ & $5,0 \mathrm{E}$ & $53,05 \mathrm{~B}$ & $0,874 \mathrm{G}$ & $21,01 \mathrm{D}$ & $14,5 \mathrm{D}$ & $40,8 \mathrm{D}$ & $4,00 \mathrm{E}$ \\
\hline 50 & Princesa & $1,25 \mathrm{E}$ & $5,5 \mathrm{~A}$ & $8,2 \mathrm{~A}$ & $52,69 \mathrm{~B}$ & $1,600 \mathrm{~F}$ & $17,47 \mathrm{H}$ & $11,8 \mathrm{~F}$ & $50,4 \mathrm{~B}$ & $4,25 \mathrm{E}$ \\
\hline 51 & LH-5 & $1,00 \mathrm{E}$ & $3,7 \mathrm{~B}$ & $4,2 \mathrm{E}$ & $55,29 \mathrm{~A}$ & $1,162 \mathrm{G}$ & $18,89 \mathrm{G}$ & $14,9 \mathrm{D}$ & $46,5 \mathrm{C}$ & $4,67 \mathrm{E}$ \\
\hline 52 & Rudá & $1,50 \mathrm{D}$ & $4,5 \mathrm{~A}$ & $6,7 \mathrm{D}$ & $50,68 \mathrm{~B}$ & $1,744 \mathrm{E}$ & $20,26 \mathrm{~F}$ & $12,8 \mathrm{E}$ & $39,1 \mathrm{E}$ & $5,40 \mathrm{D}$ \\
\hline 53 & CII-348 & $1,25 \mathrm{E}$ & $4,2 \mathrm{~A}$ & $5,5 \mathrm{E}$ & $55,45 \mathrm{~A}$ & $1,177 \mathrm{G}$ & $20,12 \mathrm{~F}$ & $13,4 \mathrm{D}$ & $41,6 \mathrm{D}$ & $4,47 \mathrm{E}$ \\
\hline 54 & $17-4-32$ & $1,00 \mathrm{E}$ & $4,7 \mathrm{~A}$ & $7,0 \mathrm{D}$ & $60,70 \mathrm{~A}$ & $1,722 \mathrm{E}$ & $22,91 \mathrm{~A}$ & $10,6 \mathrm{G}$ & $33,6 \mathrm{G}$ & $4,97 \mathrm{D}$ \\
\hline 55 & CII-285 & $1,00 \mathrm{E}$ & $4,2 \mathrm{~A}$ & $5,5 \mathrm{E}$ & $54,86 \mathrm{~A}$ & $1,656 \mathrm{E}$ & $19,93 \mathrm{~F}$ & $15,6 \mathrm{C}$ & $49,8 \mathrm{~B}$ & $5,05 \mathrm{D}$ \\
\hline 56 & CI 107-6 & $1,00 \mathrm{E}$ & $5,0 \mathrm{~A}$ & $8,0 \mathrm{~B}$ & $56,23 \mathrm{~A}$ & $1,973 \mathrm{D}$ & $21,48 \mathrm{~A}$ & $10,4 \mathrm{G}$ & $36,8 \mathrm{~F}$ & $4,72 \mathrm{E}$ \\
\hline 57 & LH-8 & $1,00 \mathrm{E}$ & $5,5 \mathrm{~A}$ & $5,5 \mathrm{E}$ & $55,78 \mathrm{~A}$ & $1,256 \mathrm{G}$ & $17,87 \mathrm{H}$ & $14,8 \mathrm{D}$ & $43,4 \mathrm{C}$ & $5,05 \mathrm{D}$ \\
\hline 58 & FT 91-4044 & $1,25 \mathrm{E}$ & $3,0 \mathrm{~B}$ & $3,5 \mathrm{E}$ & $52,42 \mathrm{~B}$ & $1,425 \mathrm{~F}$ & $20,23 \mathrm{~F}$ & $17,1 \mathrm{C}$ & $52,2 \mathrm{~B}$ & $5,10 \mathrm{D}$ \\
\hline 59 & LH-7 & $1,25 \mathrm{E}$ & $3,5 \mathrm{~B}$ & $4,5 \mathrm{E}$ & $53,80 \mathrm{~B}$ & $1,486 \mathrm{~F}$ & $24,85 \mathrm{~A}$ & $11,2 \mathrm{~F}$ & $39,2 \mathrm{E}$ & $4,87 \mathrm{E}$ \\
\hline 60 & FT 91-1535 & $1,00 \mathrm{E}$ & $5,5 \mathrm{~A}$ & $6,7 \mathrm{D}$ & $55,74 \mathrm{~A}$ & $1,851 \mathrm{D}$ & $22,62 \mathrm{~A}$ & $14,1 \mathrm{D}$ & $44,2 \mathrm{C}$ & $4,60 \mathrm{E}$ \\
\hline 61 & LH-12 & $1,00 \mathrm{E}$ & $7,0 \mathrm{~A}$ & $5,5 \mathrm{E}$ & $53,42 \mathrm{~B}$ & $1,204 \mathrm{G}$ & $19,65 \mathrm{G}$ & $16,0 \mathrm{C}$ & $37,7 \mathrm{~F}$ & $5,07 \mathrm{D}$ \\
\hline 62 & CII-122 & $1,25 \mathrm{E}$ & $3,7 \mathrm{~B}$ & $3,7 \mathrm{E}$ & $54,43 \mathrm{~B}$ & $1,579 \mathrm{~F}$ & $19,08 \mathrm{G}$ & $15,1 \mathrm{D}$ & $45,8 \mathrm{C}$ & $4,35 \mathrm{E}$ \\
\hline 63 & Pérola & $1,25 \mathrm{E}$ & $4,7 \mathrm{~A}$ & $5,2 \mathrm{E}$ & $53,28 \mathrm{~B}$ & $2,123 \mathrm{D}$ & $24,38 \mathrm{~A}$ & $11,9 \mathrm{~F}$ & $43,3 \mathrm{C}$ & $5,20 \mathrm{D}$ \\
\hline 64 & LH-10 & $1,00 \mathrm{E}$ & $4,7 \mathrm{~A}$ & $6,0 \mathrm{D}$ & $55,01 \mathrm{~A}$ & $1,613 \mathrm{~F}$ & $21,13 \mathrm{~B}$ & $15,9 \mathrm{C}$ & $50,6 \mathrm{~B}$ & $4,72 \mathrm{E}$ \\
\hline 65 & Carioca & $1,00 \mathrm{E}$ & $5,2 \mathrm{~A}$ & $8,0 \mathrm{~B}$ & $53,74 \mathrm{~B}$ & $2,173 \mathrm{D}$ & $23,75 \mathrm{~A}$ & $12,8 \mathrm{E}$ & $52,4 \mathrm{~B}$ & $5,37 \mathrm{D}$ \\
\hline 66 & FT 97-68 & $1,50 \mathrm{D}$ & $4,2 \mathrm{~A}$ & $4,7 \mathrm{E}$ & $56,85 \mathrm{~A}$ & $1,724 \mathrm{E}$ & $20,32 \mathrm{~F}$ & $15,3 \mathrm{C}$ & $60,9 \mathrm{~A}$ & $5,02 \mathrm{D}$ \\
\hline 67 & FT 97-278 & $1,50 \mathrm{D}$ & $3,5 \mathrm{~B}$ & $5,0 \mathrm{E}$ & $54,50 \mathrm{~B}$ & $2,133 \mathrm{D}$ & $21,21 \mathrm{~A}$ & $9,9 \mathrm{G}$ & $46,0 \mathrm{C}$ & $5,22 \mathrm{D}$ \\
\hline 68 & FT 97-30 & $1,25 \mathrm{E}$ & $3,7 \mathrm{~B}$ & $5,7 \mathrm{E}$ & $50,40 \mathrm{~B}$ & $1,888 \mathrm{D}$ & $18,60 \mathrm{G}$ & $11,2 \mathrm{~F}$ & $39,7 \mathrm{E}$ & $5,30 \mathrm{D}$ \\
\hline 69 & LH-13 & $1,25 \mathrm{E}$ & $3,2 \mathrm{~B}$ & $4,2 \mathrm{E}$ & $55,31 \mathrm{~A}$ & $1,799 \mathrm{E}$ & $17,74 \mathrm{H}$ & $10,8 \mathrm{G}$ & $38,9 \mathrm{~F}$ & $5,30 \mathrm{D}$ \\
\hline 70 & FT 97-30 & $1,50 \mathrm{D}$ & $4,2 \mathrm{~A}$ & $4,7 \mathrm{E}$ & $55,96 \mathrm{~A}$ & $1,336 \mathrm{G}$ & $20,52 \mathrm{~F}$ & $11,5 \mathrm{~F}$ & $42,8 \mathrm{C}$ & $5,22 \mathrm{D}$ \\
\hline 71 & FT 97-41 & $1,75 \mathrm{C}$ & $3,5 \mathrm{~B}$ & $4,7 \mathrm{E}$ & $56,95 \mathrm{~A}$ & $2,521 \mathrm{C}$ & $25,08 \mathrm{~A}$ & $11,8 \mathrm{~F}$ & $47,9 \mathrm{C}$ & $5,12 \mathrm{D}$ \\
\hline 72 & LH-9 & $1,00 \mathrm{E}$ & $4,5 \mathrm{~A}$ & $6,5 \mathrm{D}$ & $54,03 \mathrm{~B}$ & $1,527 \mathrm{~F}$ & $21,95 \mathrm{~A}$ & $14,7 \mathrm{D}$ & $54,0 \mathrm{~B}$ & $4,60 \mathrm{E}$ \\
\hline 73 & H-4-10 & $1,00 \mathrm{E}$ & $3,5 \mathrm{~B}$ & $6,2 \mathrm{D}$ & $54,86 \mathrm{~A}$ & $1,988 \mathrm{D}$ & $26,96 \mathrm{~A}$ & $11,3 \mathrm{~F}$ & $46,6 \mathrm{C}$ & $5,25 \mathrm{D}$ \\
\hline 74 & CII 164-3 & $1,00 \mathrm{E}$ & $3,0 \mathrm{~B}$ & $4,5 \mathrm{E}$ & $55,09 \mathrm{~A}$ & $1,863 \mathrm{D}$ & $21,60 \mathrm{~A}$ & $15,7 \mathrm{C}$ & $40,4 \mathrm{D}$ & $6,10 \mathrm{~B}$ \\
\hline 75 & LH-6 & $1,50 \mathrm{D}$ & $4,2 \mathrm{~A}$ & $6,0 \mathrm{D}$ & $60,78 \mathrm{~A}$ & $2,108 \mathrm{D}$ & $19,45 \mathrm{G}$ & $13,3 \mathrm{D}$ & $39,8 \mathrm{E}$ & $5,67 \mathrm{C}$ \\
\hline 76 & Aporé & $1,00 \mathrm{E}$ & $6,2 \mathrm{~A}$ & $7,7 \mathrm{C}$ & $50,47 \mathrm{~B}$ & $1,725 \mathrm{E}$ & $24,01 \mathrm{~A}$ & $10,4 \mathrm{G}$ & $43,1 \mathrm{C}$ & $5,55 \mathrm{C}$ \\
\hline 77 & CII 54 & $1,00 \mathrm{E}$ & $4,7 \mathrm{~A}$ & $7,7 \mathrm{C}$ & $53,35 \mathrm{~B}$ & $1,675 \mathrm{E}$ & $23,73 \mathrm{~A}$ & $13,5 \mathrm{D}$ & $43,4 \mathrm{C}$ & $5,05 \mathrm{D}$ \\
\hline 78 & Relav 37.19 & $1,00 \mathrm{E}$ & $4,2 \mathrm{~A}$ & $7,5 \mathrm{C}$ & $55,58 \mathrm{~A}$ & $1,560 \mathrm{~F}$ & $22,44 \mathrm{~A}$ & $17,3 \mathrm{C}$ & $49,2 \mathrm{C}$ & $5,27 \mathrm{D}$ \\
\hline 79 & R-102 & $1,00 \mathrm{E}$ & $4,5 \mathrm{~A}$ & $8,2 \mathrm{~A}$ & $58,76 \mathrm{~A}$ & $2,271 \mathrm{C}$ & $21,94 \mathrm{~A}$ & $10,9 \mathrm{G}$ & $35,9 \mathrm{G}$ & $5,07 \mathrm{D}$ \\
\hline 80 & FT 91-3057 & $1,25 \mathrm{E}$ & $4,7 \mathrm{~A}$ & $5,0 \mathrm{E}$ & $55,06 \mathrm{~A}$ & $0,929 \mathrm{G}$ & $17,36 \mathrm{H}$ & $18,2 \mathrm{~B}$ & $46,3 \mathrm{C}$ & $4,62 \mathrm{E}$ \\
\hline 81 & FT 91-4067 & $1,00 \mathrm{E}$ & $3,0 \mathrm{~B}$ & $5,5 \mathrm{E}$ & $53,68 \mathrm{~B}$ & $2,377 \mathrm{C}$ & $19,88 \mathrm{~F}$ & $8,2 \mathrm{G}$ & $34,9 \mathrm{G}$ & $5,12 \mathrm{D}$ \\
\hline 82 & R-110 & $1,50 \mathrm{D}$ & $5,0 \mathrm{~A}$ & $3,7 \mathrm{E}$ & $55,02 \mathrm{~A}$ & $1,232 \mathrm{G}$ & $19,08 \mathrm{G}$ & $17,1 \mathrm{C}$ & $52,6 \mathrm{~B}$ & $4,92 \mathrm{D}$ \\
\hline 83 & LH-3 & $1,00 \mathrm{E}$ & $4,2 \mathrm{~A}$ & $7,5 \mathrm{C}$ & $55,18 \mathrm{~A}$ & $1,746 \mathrm{E}$ & $19,26 \mathrm{G}$ & $12,7 \mathrm{E}$ & $47,4 \mathrm{C}$ & $4,95 \mathrm{D}$ \\
\hline 84 & CII-74 & $1,25 \mathrm{E}$ & $4,5 \mathrm{~A}$ & $7,7 \mathrm{C}$ & $56,54 \mathrm{~A}$ & $1,746 \mathrm{E}$ & $20,90 \mathrm{E}$ & $11,7 \mathrm{~F}$ & $33,8 \mathrm{G}$ & $4,02 \mathrm{E}$ \\
\hline 85 & FT 206 & $1,00 \mathrm{E}$ & $3,0 \mathrm{~B}$ & $5,2 \mathrm{E}$ & $51,93 \mathrm{~B}$ & $2,221 \mathrm{D}$ & $23,51 \mathrm{~A}$ & $16,3 \mathrm{C}$ & $56,0 \mathrm{~B}$ & $6,35 \mathrm{~A}$ \\
\hline 86 & ESAL 550 & $2,25 \mathrm{~B}$ & $2,2 \mathrm{~B}$ & $2,7 \mathrm{E}$ & $54,18 \mathrm{~B}$ & $1,488 \mathrm{~F}$ & $32,15 \mathrm{~A}$ & $11,0 \mathrm{~F}$ & $28,2 \mathrm{G}$ & $3,47 \mathrm{E}$ \\
\hline 87 & CII 103 & $2,75 \mathrm{~A}$ & $4,2 \mathrm{~A}$ & $4,5 \mathrm{E}$ & $56,22 \mathrm{~A}$ & $1,913 \mathrm{D}$ & $22,22 \mathrm{~A}$ & $8,9 \mathrm{G}$ & $31,5 \mathrm{G}$ & $4,40 \mathrm{E}$ \\
\hline 88 & CI 102 & $1,25 \mathrm{E}$ & $4,2 \mathrm{~A}$ & $6,7 \mathrm{D}$ & $55,46 \mathrm{~A}$ & $2,347 \mathrm{C}$ & $24,34 \mathrm{~A}$ & $12,3 \mathrm{~F}$ & $37,0 \mathrm{~F}$ & $4,75 \mathrm{E}$ \\
\hline 89 & ESAL 693 & $1,50 \mathrm{D}$ & $5,2 \mathrm{~A}$ & $3,5 \mathrm{E}$ & $53,35 \mathrm{~B}$ & $0,986 \mathrm{G}$ & $24,69 \mathrm{~A}$ & $11,7 \mathrm{~F}$ & $38,4 \mathrm{~F}$ & $3,77 \mathrm{E}$ \\
\hline \multirow[t]{2}{*}{90} & Carioca Precoce & $1,00 \mathrm{E}$ & $3,5 \mathrm{~B}$ & $5,7 \mathrm{E}$ & $53,25 \mathrm{~B}$ & $2,522 \mathrm{C}$ & $24,73 \mathrm{~A}$ & $9,7 \mathrm{G}$ & $45,4 \mathrm{C}$ & $5,45 \mathrm{C}$ \\
\hline & Média & 1,19 & 4,39 & 5,7 & 54,50 & 1,802 & 21,08 & 12,69 & 42,4 & 5,00 \\
\hline
\end{tabular}

* Genótipos com médias não seguidas da mesma letra diferem, em nível de 5\% de probabilidade de erro, pelo teste de Scott-Knott. 


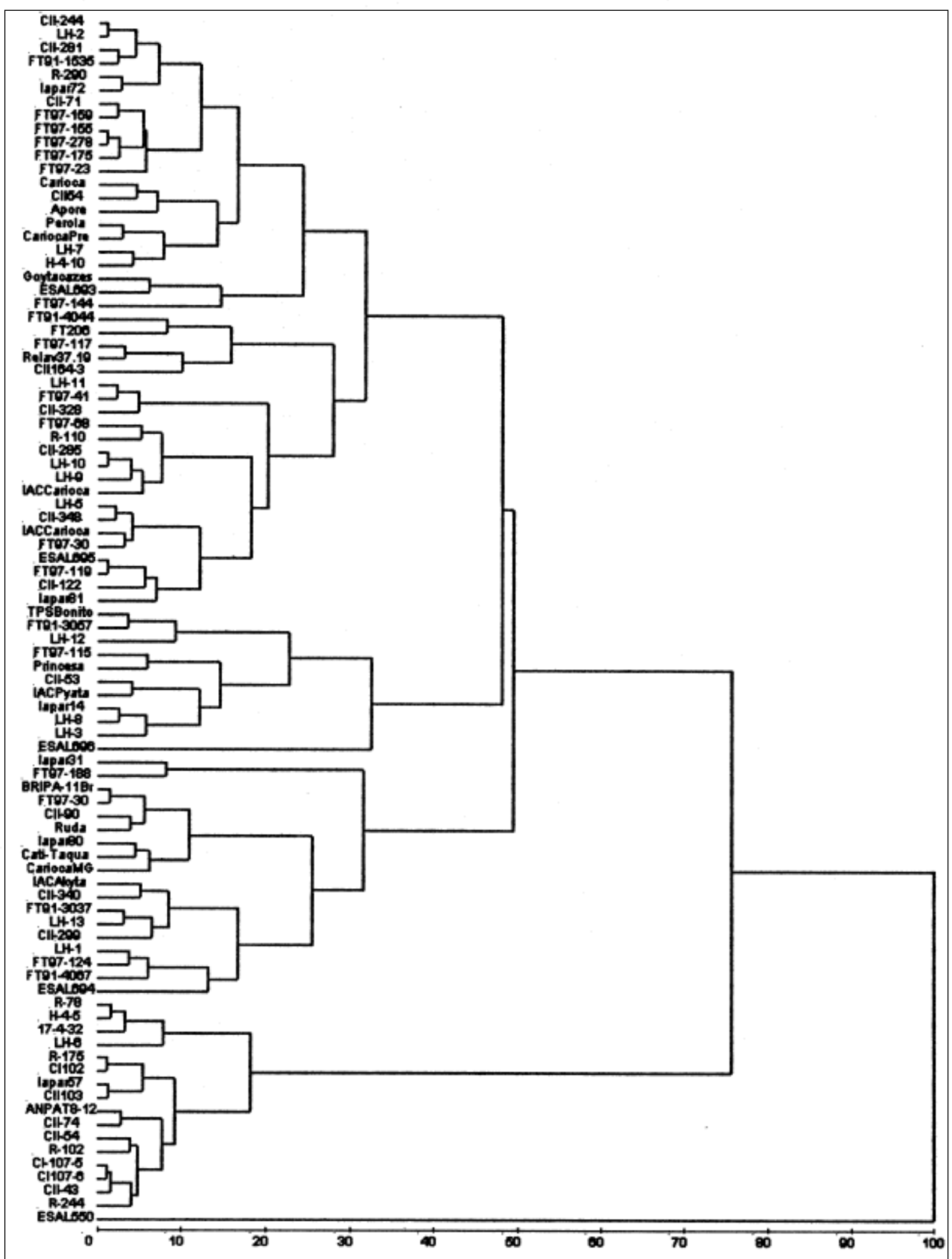

Figura 1 - Dendrograma de dissimilaridade obtido pelo método hierárquico da ligação completa, baseado nas distâncias generalizadas de Mahalanobis entre 90 genótipos de feijão carioca. Santa Maria, UFSM, 2000.

Ciência Rural, v. 33, n. 3, mai-jun, 2003. 
em rendimento de grãos, sendo que essa apresenta várias outras características de destaque, como a coloração clara do tegumento das sementes, maior massa de 100 sementes e número de sementes por planta.

Procedendo à análise dos resultados dos grupos de dissimilaridade, verifica-se que a formação de três grandes grupos pode ser obtida com distâncias próximas a $75 \%$ entre os mesmos (Figura 1). O grupo 1 (G1) constituído pelo genótipo ESAL 550, na parte inferior da figura. O grupo $1(\mathrm{G} 2)$ constituído por 16 genótipos, R-244 até R-78 (do segundo até o décimo sétimo, de baixo para cima); e, o grupo 3 (G3), pelos demais 73 genótipos (ESAL 694 até CII-244). Como as hibridações promissoras devem ser planejadas entre genótipos dos três grupos dissimilares, ter-se-á que identificar, dentro de cada grupo, os genótipos com características que visam a complementar a formação da linhagem a ser obtida. O genótipo ESAL 550, única do grupo G1, tem como característica favorável o tamanho das sementes (maior massa de cem sementes). No grupo G2, os genótipos LH-6, 17-4-32, R-78, H-4-5 e R-102 possuem a coloração clara do tegumento das sementes (característico de feijão recém-colhido) que é uma característica de grande importância em programas de melhoramento de feijão carioca, pois quanto maior o valor de 'L', maior será a claridade do tegumento das sementes e, conseqüentemente, maior será sua aceitação comercial.

No grupo G3, os genótipos com destaque na produtividade de grãos (classes $\mathrm{A}, \mathrm{B}$ e $\mathrm{C}$ do teste de médias) em ordem decrescente são: FT 97-188, CatiTaquari, CII-328, Carioca Precoce, FT 97-41, LH-11, FT 91-4067, Iapar 31, CI 102, Carioca MG, CII-54 e R-102. Além do rendimento, o genótipo FT 97-188 têm características de altura de inserção de primeiro e de último legume favoráveis à colheita mecânica e os genótipos FT 97-188, Cati Taquari, Carioca Precoce, Iapar 31, Carioca MG e CII-54 têm maior número de sementes por legume.

\section{CONCLUSÕES}

Os caracteres incidência de ferrugem nos legumes, acamamento, nota geral, cor do tegumento ("L"), rendimento de grãos, massa de 100 sementes, altura de inserção do primeiro legume, altura de inserção do último legume e número de sementes por legume são os fatores que mais contribuem para a divergência genética entre os genótipos de feijão carioca.

Populações segregantes com variabilidade genética superior, podem ser obtidas com hibridações entre o genótipo ESAL 550 com genótipos do grupo 2 (LH-6, 17-4-32, R-78, H-4-5 e R-102) e/ou com genótipos do grupo 3 (FT 97-188, Cati-Taquari, CII-328, Carioca Precoce, FT 97-41, LH-11, FT 91-4067, Iapar 31, CI 102, Carioca MG, CII-54 e R-102).

\section{REFERÊNCIAS BIBLIOGRÁFICAS}

ABREU, A.F.B. et al. Progresso do melhoramento genético do feijoeiro nas décadas de setenta e oitenta, nas regiões sul e Alto Paranaíba em Minas Gerais. Pesquisa Agropecuária Brasileira, Brasília, v.29, n.1, p.105-112, 1994.

CEPEF. Feijão: recomendações técnicas para cultivo de feijão no Rio Grande do Sul. Santa Maria : PALLOTTI, 2000. 80p.

CIAT. Standard system for the evaluation of bean germplasm. Cali : CIAT, 1987. 54p.

CARNEIRO, G.E.S.; SOARES, D.M.; COSTA, J.G.C. Resultados do ensaio Sul-Brasileiro de avaliação de linhagens de feijão nos anos 1997/98 e 1998/99. Santo Antônio de Goiás : Embrapa Arroz e Feijão, 2000. 77p. (Embrapa Arroz e Feijão. Documentos, 102).

COIMBRA, J.L.M.; CARVALHO, F.I.F. Divergência genética em linhagens de feijão preto (Phaseolus vulgaris L.) preditas através de variáveis quantitativas. Revista Científica Rural, Bagé, v.4, n.1, p.47-53, 1999.

COIMBRA, J.L.M. et al. Divergência genética em feijão preto. Ciência Rural, Santa Maria, v.29, n.3, p.427-431, 1999.

COIMBRA, J.L.M. et al. Correlações canônicas: II - Análise do rendimento de grãos de feijão e seus componentes. Ciência Rural, Santa Maria, v.30, n.1, p.31-35, 2000.

CRUZ, C.D. Programa GENES: versão windows: aplicativo computacional em genética e estatística. Viçosa : UFV, 2001. 648p.

CRUZ, C.D., REGAZZI, A.J. Modelos biométricos aplicados ao melhoramento genético. Viçosa : UFV, 1997. 390 p.

FONSECA, J.R.; SILVA, H.T. Emprego da análise multivariada na caracterização de acessos de feijão (Phaseolus vulgaris L.). Revista Brasileira de Sementes, Campinas, v.19, n.2, p.335-341, 1997.

FONSECA, J.R.; SILVA, H.T. Identificação de acessos de feijão por meio de técnicas multivariadas. Pesquisa Agropecuária Brasileira, Brasília, v.34, n.3, p.409-414, 1999.

MACHADO, C.F.; SANTOS, J.B.; NUNES, G.H.S. Escolha de genitores de feijoeiro por meio da divergência avaliada a partir de caracteres morfo-agronômicos. Bragantia, Campinas, v.59, n.1, p.11-20, 2000.

McCLEAN, P.E.; MYRES, J.M.; HAMMOND, J.J. Coefficient of parentage and cluster analysis of north American dry bean cultivars. Crop Science, Madison, v.33, n.1, p.190-193, 1993. 
RAMALHO, M.A.P.; FERREIRA, D.F.; OLIVEIRA, A.C. Experimentação em genética e melhoramento de plantas. Lavras : UFLA, 2000. 326p.

RIBEIRO, N.D. Escolha de genitores de feijoeiro por meio da divergência genética. Santa Maria, 2001. 80p. Tese (Doutorado em Agronomia) - Programa de Pós-
Graduação em Agronomia, Universidade Federal de Santa Maria.

ROLAS. Recomendação de adubação e calagem para os estados do Rio Grande do Sul e Santa Catarina. 3. ed. Passo Fundo : SBCS, 1995. 223p. 